\title{
Características generales de la osteomielitis en pacientes tratados con implantes dentales
}

\author{
General characteristics of osteomyelitis in patients treated with \\ dental implants
}

Brito Falcón-Guerrero (1) ${ }^{1 a}$

\begin{abstract}
Resumen
La osteomielitis de los maxilares es un proceso inflamatorio relativamente raro dentro del campo de la implantología y para caracterizar a esta patología es necesario analizar no solo al propio paciente sino también las características clínicas, antecedentes patológicos, la etiología y el tratamiento; con la intención de lograr un diagnóstico precoz y así evitar la pérdida prematura de los implantes dentales. El objetivo de esta revisión es describir las características generales de la osteomielitis en pacientes tratados con implantes dentales. Concluyendo en que las características generales encontradas pueden servir de apoyo para un diagnóstico precoz de la osteomielitis en relación a los implantes dentales.
\end{abstract}

Palabras Clave: Implantes dentales; Infección; Maxilar; Osteomielitis; Factores de riesgo. (Fuente: DeCS BIREME)

\begin{abstract}
Osteomyelitis of the jaws is a relatively rare inflammatory process within the field of implantology and to characterize this pathology it is necessary to analyze not only the patient himself but also the clinical characteristics, pathological antecedents, etiology and treatment; with the intention of achieving an early diagnosis and thus avoiding the premature loss of dental implants. The objective of this review is to describe the general characteristics of osteomyelitis in patients treated with dental implants. Concluding that the general characteristics found can serve as support for an early diagnosis of osteomyelitis in relation to dental implants.
\end{abstract}

Key words: Dental implants; Infection; Maxilla; Osteomyelitis; Risk factors. (Source: MeSH NLM)

Federación Iberopanamericana de Periodoncia (FIPP).Lima, Perú. ${ }^{2}$ Asociación Peruana de Periodoncia y Oseointegración (APPO). Lima, Perú

a PhD. Cirujano Dentista, Especialista de periodoncia

Correspondencia:

Brito Falcón Guerrero

Calle Zela 747. Tacna, Perú.

Correo electrónico: artdent2000@hotmail.com
Este es un artículo Open Access distribuido bajo la licencia Creative Commons Atribución 4.0 Internacional (CC BY 4.0)

https://creativecommons.org/licenses/by/4.0/deed.es

Citar como: Falcón Guerrero B. Características generales de la osteomielitis en pacientes tratados conimplantes dentales. KIRU. 2021;18(1):65-69. https://doi.org/10.24265/kiru. 2021.v18n1.09. 


\section{INTRODUCCIÓN}

Se considera que un implante dental (ID) tiene una tasa de supervivencia acumulada del $87,8 \%$ después de 36 años de seguimiento ${ }^{(1)}$, sin embargo esta sobrevida depende de que se realice una cirugía atraumática, que no se presente infección o existan factores de riesgo como enfermedades metabólicos; destacando la infección temprana $u$ osteomielitis como causa principal de la perdida temprana del ID (2).

La osteomielitis es una infección del hueso y médula ósea, acompañada de un proceso destructivo gracias a que posee un pobre sistema vascular que no permite una buena respuesta de defensa y del mismo modo los antibióticos no llegan de forma adecuada a la zona de infección ${ }^{(3)}$. Se presenta después de un traumatismo o de una cirugía ósea o por consecuencia de una insuficiencia vascular del tejido óseo, y se presenta con más frecuencia en las áreas dentadas ${ }^{(4)}$, y en su forma aguda es supurativa y compromete la respuesta vascular de la médula y el periostio, produciendo una destrucción que conduce a la muerte del tejido, ocasionando que se instale la forma crónica ${ }^{(3)}$.

El pronóstico de la osteomielitis depende del mecanismo de infección, la virulencia del agente infeccioso, el estado inmunológico y los antecedentes comórbidos que posea el paciente. Describiéndose tres tipos de osteomielitis (Os) según la fuente de la infección: 1) Os secundaria a un foco contiguo de infección (después de un traumatismo, cirugía o inserción de una articulación protésica); 2) Os secundaria asociada con insuficiencia vascular, como en pacientes con diabetes mellitus y enfermedad vascular periférica; y 3) Os posterior a una invasión vascular de un proceso infeccioso $(3,5)$

Ante el aumento de la demanda del Implante Dental hay un incremento progresivo del número de casos de Os asociados con implantes dentales ${ }^{(6)}$. En la etapa inicial del implante dental se pueden presentar infecciones durante el primer mes, lo que aumenta el riesgo de falla temprana en un 2,4 $\%$ sin que estos hayan entrado en función ${ }^{(7-9)}$. La Os en los maxilares son raras gracias a una mejor higiene bucal, el uso de antibióticos y un diagnóstico temprano ${ }^{(10-12)}$; sin embargo, existe un subtipo agresivo que requiere un tratamiento más amplio y complejo (13). Presentándose preocupación por la perdida temprana del Implante, por lo que es necesario tener en cuenta todos los principios quirúrgicos para reducir el riesgo de la presencia de la Os ${ }^{(11)}$. Ante esta situación, se hace necesario establecer un buen diagnóstico por lo que se necesita actualizar los aspectos relacionados con la Os en pacientes tratados con implantes dentales; enfatizando en las características relacionadas a esta enfermedad y el riesgo que representa, teniendo en cuenta que se presenta de preferencia en una población adulta mayor. Es por ello, que el objetivo de la presente revisión es describir las características generales de la osteomielitis en pacientes tratados con implantes dentales.

\section{CARACTERÍSTICAS SOCIO DEMOGRÁFICAS Y UBICACIÓN DE LA OSTEOMIELITIS}

Dentro de estas características se evalúa la edad y el sexo de la población que presento el cuadro de osteomielitis relacionada a los Implantes Dentales. Se han descrito reportes como el de Kelleserian et al ${ }^{(5)}$, quienes encontraron que la osteomielitis es más frecuente en pacientes mujeres en comparación con los hombres en una proporción de 2:1, y presentaban una media de 60,26 años de edad. Del mismo modo, Yahomlam et al ${ }^{(13)}$, reportan una mayoría de casos en mujeres en un $71,5 \%$, con una media de edad de 59 años.

Chatelain et al. ${ }^{(14)}$, manifiestan que las mujeres representaban el $100 \%$ de los casos de Os reportados, con una media de 59,8 años de edad; así mismo, ShnaidermanShapiro et al. ${ }^{(15)}$, mencionan un $80 \%$ de prevalencia en mujeres y 64,8 años de edad y finalmente Semel et al. ${ }^{(11)}$, en una serie de casos hallaron que la prevalencia en mujeres 
era de $60 \%$ con una media de 59,8 años de edad.

Sobre la ubicación en los maxilares, se identifica una mayor predisposición de que la mandíbula sea la más afectada, solo se ha encontrado un caso de Os relacionada a IDs que afecta al maxilar superior ${ }^{(13)}$. Esto se puede deber a las características propias del tejido óseo de este maxilar inferior, donde existe una pobre vasculatura terminal, su estructura corticoesponjosa y la baja cobertura mucosa, son argumentos a favor de que la mandíbula sea el sitio preferido de Os ${ }^{(12)}$. Por lo que, la enfermedad puede ser ligada en pacientes a partir de 60 años de edad, con mayor predilección en las mujeres y del maxilar inferior.

\section{TIPOS DE OSTEOMIELITIS, ANTECEDENTES PATOLÓGICOS Y ETIOLOGÍA}

Sobre el tipo de osteomielitis diagnosticadas, los distintos estudios analizados presentan 13 cuadros crónicos ${ }^{(10,11,15-20)}, 9$ osteomielitis agudas ${ }^{(11,14,21)}$; evidenciando que no hay una mayor diferencia entre los diagnósticos agudos y crónicos; resaltando que en los cuadros agudos todos los Implantes dentales fueron perdidos, mientras que cuando el tipo fue crónico se llegaron a conservar algunos Implantes.

Respecto a los antecedentes patológicos 15 pacientes estaban sanos (sin historial médico), y 7 tenían antecedentes de ser fumadores, 6 son hipertensos, 5 son diabéticos, 2 sufren de asma, 2 de hipotiroidismo, 2 con Hipercolesterolemia, 1 con bronquitis crónica y pirosis, 1 con neutropenia leve, 1 con desorden bipolar, 1 con síndrome de sinovitis, acné, pustulosis, hiperostosis y osteítis (SAPHO), pustulosis palmoplantar y osteosclerosis de la clavícula, 1 con osteoporosis, la que trata con bifosfonatos; y 1 presentaba Osteopetrosis. (10, 11,14-21). Por lo tanto, la presencia de la Os no necesariamente estaría relacionada a un antecedente patológico, sino que puede estar relacionado a algún factor local contaminante durante el procedimiento quirúrgico de instalación de los implantes dentales.
Dentro de la etiológia se halló los siguientes agentes microbiológicos familia de estreptococos: 6 casos de Estreptococos anginosus, 1 caso de Estreptococos epidermidis, 2 casos de Estreptococos intermedius, 1 caso de Estreptococos $b$ hemolitico. También se reporta 14 casos donde en la mayoría se encontró Actinomices, y Enterobacteria cloacae, 1 caso de E-Coli, 1 caso de Veillonella atypical y 2 casos de Cándida (10,11,14-21). Cabe destacar que la osteomielitis fúngica no es infrecuente y se da en pacientes médicamente comprometidos posiblemente debido a una respuesta inmunológica deficiente y puede conducir a una gran destrucción del hueso maxilar ${ }^{(22)}$. Pero también se debe considerar que existe una osteomielitis no bacteriana, como la osteomielitis multifocal crónica recurrente, que es un trastorno inflamatorio raro y no infeccioso que causa lesiones óseas líticas multifocales, donde se dan periodos de exacerbación y remisión periódica ${ }^{(23)}$.

\section{CARACTERÍSTICAS CLÍNICAS Y TRATAMIENTO}

Los pacientes presentaron en general los siguientes signos y síntomas clínicos: fiebre, inflamación, dolor, trismus, edema facial, supuración, desensibilización, parestesia y neuropatía del nervio alveolar inferior y formación de abscesos en el espacio profundo del cuello del cuello, Inflamación gingival y peri implantaría. ${ }^{(10,11,14-21)}$. Aparte se encontraron pruebas radiográficas de imágenes radiolúcidas difusas, que muestran anomalías en la estructura ósea y evidencia la presencia de Os; sin embargo, Shin et al (16). menciona un único caso, que presento una imagen radiopaca bien definida.

Como estos cuadros de Os tienen una variada causa de microrganismos, existen varias alternativas de tratamiento que inician siempre con un adecuado manejo farmacológico. Semel et al ${ }^{(13)}$, manifiestan que para el tratamiento, se requiere una antibiótico-terapia prolongada y en altas dosis, junto con un tratamiento quirúrgico agresivo, para el tratamiento de la osteomielitis de los maxilares. Coincidiendo 
con los resultados de esta revisión, donde los tiempos de administración antibiótica van desde menos de 1 mes ${ }^{(10,13,14,21)}$ a más de 24 meses ${ }^{(13,20)}$ de cobertura antibiótica, optando por el uso de una variedad de antibióticos para anaerobios y Gram (-), como: Clindamicina, Ciprofloxacino, Amoxicilina mas ac. Clavulánico, Levofloxacina, Rifampicina y Claritromicina mas Levofloxacina (10,11,14-21), enfatizando en la resistencia bacteriana a la penicilina $^{(14)}$.

Como alternativa, Sun et al. ${ }^{(24)}$, describen el efecto del sulfato de calcio impregnado con vancomicina, despues de realizar un desbridamiento quirúrgico para la osteomielitis crónica mostrando buenos resultados, además, el sulfato de calcio puede promover la formación de hueso nuevo. Este manejo antibiótico va de la mano con el tratamiento quirúrgico que consiste desde el retiro del implante, curetaje, drenaje extraoral, secuestrectomía y tratamiento con oxígeno hiperbárico; hasta tratamientos más radicales como la Hemimandibulectomía y reconstrucción con injerto de hueso ilíaco ${ }^{(10,}$ $11,14-21,25)$

Dentro de estas alternativas, el oxígeno hiperbárico está demostrando ser un buen complemento para el tratamiento de Os e IDs (26). Si se presentara fractura mandíbular se puede manejar con una reducción cerrada, fijación rígida mediante placas de osteosíntesis e injertos óseos. Sin embargo, pueden presentarse complicaciones, como Os sobre agregada, pseudoartrosis, fractura de la placa, aflojamiento de tornillos y dehiscencias con infecciones posteriores ${ }^{(27)}$.

\section{CONCLUSIONES}

Las características generales encontradas en esta revisión pueden servir de apoyo para un diagnóstico precoz de la osteomielitis en relación a los implantes dentales.

Fuente de financiamiento: Autofinanciado.

Conflicto de intereses: El autor declaró no tener conflictos de interés.

\section{REFERENCIAS BIBLIOGRÁFICAS}

1. Chrcanovic BR, Kisch J, Albrektsson T, Wennerberg A. A retrospective study on clinical and radiological outcomes of oral implants in patients followed up for a minimum of 20 years. Clin Implant Dent Relat Res. 2018 Apr;20(2):199-207. doi: 10.1111/cid.12571.

2. Fathima M, Sinha N, Ali SM. Failures in Dental Implants: A Review. Int J Adv Health Sci. 2017;4(2):5-9. Disponible en: https://www.researchgate.net/publication/32113 5267_Failures_in_Dental_Implants_A_Review

3. Gomes D, Pereira $\bar{M}$, Bettencourt AF. Osteomyelitis: an overview of antimicrobial therapy. Brazilian Journal of Pharmaceutical Sciences. 2013; 49(1):13-27. Disponible en: http://www.scielo.br/scielo.php?script=sci_artte xt\&pid=S1984-82502013000100003

4. Dym H, Zeidan J. Microbiology of Acute and Chronic Osteomyelitis and Antibiotic Treatment. Dent Clin North Am. 2017 Apr;61(2):271-282. doi: 10.1016/j.cden.2016.12.001. PMID: 28317566.

5. Surdu-Bob CC, Coman C, Barbuceanu F, Turcu D, Bercaru N, Badulescu M. The influence of foreign body surface area on the outcome of chronic osteomyelitis. Medical Engineering and Physics. 2016;38(9):870-876. Disponible en: https://www.ncbi.nlm.nih.gov/pubmed/2726423 $\underline{9}$

6. Kellesarian SV, Javed F, Romanos GE. Osteomyelitis Arising Around Osseointegrated Dental Implants: A Systematic Review. Implant Dent. 2018; 27(2): 226-235. Disponible en: https://www.researchgate.net/publication/32275 5813 Osteomyelitis_Arising_Around_Osseoint egrated_Dental_Implants_A_Systematic_Revie w

7. Camps-Font O, Martín-Fatás P, Clé-Ovejero A, Figueiredo R, Gay-Escoda C, ValmasedaCastellón E. Postoperative infections after dental implant placement: variables associated with increased risk of failure. J Periodontol. 2018 Oct;89(10):1165-1173.

8. Figueiredo $R$, Camps-Font $O$, ValmasedaCastellón E, Gay-Escoda C. Risk Factors for Postoperative Infections After Dental Implant Placement: A Case-Control Study. J Oral Maxillofac Surg. 2015 Dec;73(12):2312-8. Disponible en: https://www.sciencedirect.com/science/article/a bs/pii/S0278239115010642

9. Camps-Font O, Figueiredo R, ValmasedaCastellón E, Gay-Escoda C. Postoperative Infections After Dental Implant Placement: Prevalence, Clinical Features, and Treatment. Implant Dent. 2015;24(6):713-719.

10. Schlund M, Raoul G, Ferri J, Nicot R. Mandibular osteomyelitis following implant placement. J Oral Maxillofac Surg. 2017 Dec;75(12):2560.e1-2560.e7. Disponible en: 
https://www.joms.org/article/S02782391(17)31011-X/fulltext

11. Semel G, Wolff A, Shilo D, Akrish S, Emodi O, Rachmiel A. Mandibular osteomyelitis associated with dental implants. A case series. Eur J Oral Implantol. 2016;9:435-442.

12. Andre C-V, Khonsari RH, Ernenwein D, Goudot P, Ruhin B. Osteomyelitis of the jaws: A retrospective series of 40 patients. J Stomatol Oral Maxillofac Surg. 2017;118(5):261-264. Disponible en: https://www.sciencedirect.com/science/article/a bs/pii/S246878551730085X?via\%3Dihub

13. Yahalom R, Ghantous Y, Peretz A, Abu-Elnaaj I. The possible role of dental implants in the etiology and prognosis of osteomyelitis: A retrospective study. Int $\mathrm{J}$ Oral Maxillofac Implants. 2016;31:1100-1109.

14. Chatelain S, Lombardi T, Scolozzi P. Streptococcus anginosus dental implant related osteomyelitis of the jaws: An insidious and calamitous entity. J Oral Maxillofac Surg. 2018 ;76(6):1187-1193. Disponible en: https://www.joms.org/article/S02782391(18)30032-6/fulltext

15. Shnaiderman-Shapiro A, Dayan D, Buchner A, Schwartz I, Yahalom R, Vered M. Histopathological spectrum of bone lesions associated with dental implant failure: Osteomyelitis and beyond. Head Neck Pathol. 2015;9:140-146.

16. Shin HS, Kim BC, Lim HJ, Jo SY, Lee J. Chronic osteomyelitis induced by the placement of dental implants on cementoosseous dysplasia. $\mathrm{Br} \mathrm{J}$ Oral Maxillofac Surg (2019), 57(3):268-270. Disponible en: https://www.bjoms.com/article/S02664356(19)30018-X/fulltext

17. Sakai K, Shibui T, Sato K, Hashimoto K, Ushioda T, Seta N, Takahashi S, Nomura T. Mandibular osteomyelitis related to SAPHO syndrome following dental implant surgery: A case report. Journal of Oral and Maxillofacial Surgery, Medicine, and Pathology. 2018; 30(5):452-456.

18. Schlund M, Raoul G, Ferri J, Nicot R. Mandibular osteomyelitis following implant placement. J Oral Maxillofac Surg. 2017 Dec;75(12):2560.e1-2560.e7. Disponible en: https://www.joms.org/article/S02782391(17)31011-X/fulltext

19. Balanger M, Hinet $M$, Vacher $C$, Bellaiche $N$, Charrier JL, Millot S. Osteomyelitis of the
Mandible after Dental Implants in an Immunocompetent Patient. Case Reports in Dentistry; 2017. Article ID 9525893, 5 pages. doi:

https://doi.org/10.1155/2017/9525893.

Disponible

en:

https://www.hindawi.com/journals/crid/2017/95 25893/

20. Naval L, Molini MS, Herrera G, Naval B. Dental implants and osteomyelitis in a patient with osteopetrosis. Quintessence Int. 2014;45:765768.

21. Doll C, Hartwig S, Nack C, Nahles S, Nelson $\mathrm{K}$, Raguse JD. Dramatic course of osteomyelitis in a patient treated with immediately placed dental implants suffering from uncontrolled diabetes: A case report. Eur J Oral Implantol. 2015; 8:405-410.

22. Balaji SM, Balaji P. Maxillary rehabilitation after complete destruction by fungal osteomyelitis. Indian J Dent Res. 2019 Sep-Oct;30(5):807809. doi: 10.4103/ijdr.IJDR_711_19. Disponible en: http://www.ijdr.in/article.asp?issn=09709290; year $=2019 ;$ volume $=30 ;$ issue $=5 ;$ spage $=80$ 7; epage $=809$; aulast $=$ Balaji

23. Gicchino MF, Diplomatico M, Granato C, Capalbo D, Marzuillo P, Olivieri AN, et al. Chronic recurrent multifocal osteomyelitis: a case report. Ital J Pediatr. 2018; 44(1):26.

24. Sun HJ, Xue L, Wu CB, Zhou Q. Use of Vancomycin-Impregnated Calcium Sulfate in the Treatment of Osteomyelitis of the Jaw. J Oral Maxillofac Surg. 2017 Jan;75(1):119-128. doi: 10.1016/j.joms.2016.06.178.

25. Patel SY, Kim DD, Ghali GE. Maxillofacial Reconstruction Using Vascularized Fibula Free Flaps and Endosseous Implants. Oral Maxillofac Surg Clin North Am. 2019 May;31(2):259-284.

doi: 10.1016/j.coms.2018.12.005.

26. Re K, Patel S, Gandhi J, Suh Y, Reid I, Joshi G, Smith NL, Khan SA. Clinical utility of hyperbaric oxygen therapy in dentistry. Med Gas Res. 2019 Apr-Jun;9(2):93-100. doi: 10.4103/2045-9912.260651.

27. Soehardi A, Meijer GJ, Manders R, Stoelnga PJ. An inventory of mandibular fractures associated with implants in atrophic edentulous mandibles: a survey of Dutch oral and maxillofacial surgeons. Int J Oral Maxillofac Implants. 2011 Sep-Oct;26(5):1087-93.

Britto Falcón-Guerrero

ORCID iD: (1) https://orcid.org/0000-0002-9585--7052

artdent2000@hotmail.com

Copyright (C) La revista. La revista Kiru es publicada por la Facultad de Odontología de la Universidad de San Martín de Porres, en Lima, Perú. 